\title{
Ideas, creencias y percepciones acerca de la salud. Reseña histórica
}

\author{
Leopoldo Vega-Franco, MSP, MSc.(1)
}

\begin{abstract}
Vega-Franco L.
Ideas, creencias y percepciones acerca de la salud. Reseña histórica.

Salud Publica Mex 2002:44:258-265. El texto completo en inglés de este artículo está disponible en: http://www.insp.mx/salud/index.html

\section{Resumen}

Con el objeto de tener una aproximación más universal al concepto de salud se analizan las ideas, creencias y percepciones documentadas en la historia de las culturas dominantes en el mundo oriental y occidental. Se exponen las contribuciones de filósofos, médicos y pensadores, respecto a las sustancias, fuerzas y principios biológicos elementales del organismo que intervienen en la vida y el destino del hombre. El análisis de estas contribuciones permite reconocer que en diversas culturas se ha definido la salud con términos cuyo significado equivale a las palabras: equilibrio, armonía, equidad, mientras la enfermedad se ha descrito con los antónimos de estos vocablos. Se piensa que la teoría ecológica de la "resilencia", para estudiar la capacidad de adaptación de ecosistemas, puede orientar las ideas en investigaciones fisiológicas de los procesos que conducen al organismo a una nueva condición estable con el medio externo, para conocer el margen impreciso que separa la salud de la enfermedad. El texto completo en inglés de este artículo está disponible en: http://www.insp.mx/salud/ index.html
\end{abstract}

Palabras clave: concepto de salud; la salud en la historia; filosofía de la salud

\author{
Vega-Franco L. \\ Ideas, beliefs and perceptions \\ about health. A historical account. \\ Salud Publica Mex 2002;44:258-265. \\ The English version of this paper \\ is available at: http://www.insp.mx/salud/index.html
}

\begin{abstract}
A bstract
To get a closer approach to the concept of health, this paper reviews the ideas, beliefs, and perceptions about health that have been accepted by Eastern and W estern cultures. H ealth conceptions by philosophers, physicians, and thinkers are presented, particularly as they relate to substances, forces, and elementary biological principles of the human body that determine human life and fate. Analysis of these conceptions makes it possible to recognize different definitions of health as a status of balance, harmony, and equity. $0 \mathrm{n}$ the contrary, disease has been defined with the antonyms of health words. The ecolo gical theory of "resilience" may be useful to analyze the adaptive capacity of ecosystems and to suggest ideas for physiological research on processes leading the human body to a new status of balance with the external environment, and thus define the blurring boundaries between health and disease.
\end{abstract}

Key words: health concept; health in history; health philosophy

Fecha de recibido: 15 de junio de 2001 - Fecha de aprobado: 2 de abril de 2002 Solicitud de sobretiros: D r. Leopoldo Vega-Franco. Magdalena 408, Colonia Del Valle 03100, D elegación B. Juárez, México D.F., México. Correo electrónico: Ivegaf@msn.com 
Cuando la salud está ausente, la sabiduría no puede revelarse, el arte no se manifiesta, la fuerza no lucha, el bienestar es inútil y la inteligencia no tiene aplicación.

Herófilo de Cos (382-322 a. C.)

$\mathrm{E}$ s común que la gente defina la salud como la ausencia de enfermedad: como si en ella estuviera arraigada la idea de una vivencia fugaz dentro de una secuencia continua de padecimientos, o bien que su percepción fuese resabio de experiencias colectivas de épocas pretéritas. Se antoja pensar que la gente siente la salud de la misma manera que algunas personas advierten la libertad al ser sometidas a la esclavitud. No en vano Henri-Francois Becque ${ }^{1}$ opina que "La libertad y la salud son condiciones semejantes: el hombre sólo percibe su presencia cuando están ausentes". En cambio, quienes reflexionan acerca de su entorno y expresan los sentimientos de su mundo interior, definen la salud por el desmedro de sus capacidades, como lo hizo Herófilo ${ }^{2}$ hace poco más de 25 siglos. Tal parece que la capacidad de sentir y razonar acerca del ambiente y las circunstancias que transcurren en nuestra vida cotidiana permiten dar a esta palabra un significado diferente, aunque esta apreciación puede ser sólo una interpretación parcial y personal de lo que para algunos es un proceso interminable de enfermedad que se alterna con espejismos de salud.

Han sido muchos los que han definido el concepto de salud o han expresado alguna opinión acerca de este atributo, pero tal vez han sido más los que se han ocupado en restarles valor a estos juicios. En lo que probablemente todos han coincidido -los que han expresado alguna definición, los que disienten de éstas y los que callan su opinión- es en el viejo anhelo latino de tener una mente sana en un cuerpo sano (mens sana in corpore sano). Cabe pues precisar el sentido que se ha dado a algunas de las palabras que se han usado al definirla.

Se puede afirmar que en todas las lenguas hay vocablos afines a lo que en nuestro idioma se entiende como bienestar. En este fonema se resume la compleja percepción de quienes disfrutan una vida cómoda, con sus necesidades básicas satisfechas y en buen estado físico, por lo que esta palabra también se usa para expresar el concepto que implica "estar sano". Con esta connotación un grupo de expertos de la Organización Mundial de la Salud (OMS) definió la salud hace poco más de 50 años como: "el completo estado de bienestar físico, mental y social y no sólo la ausencia de enfermedad o invalidez". No se puede negar que en esta proposición los elementos del discurso que traducen salud son trasladados al concepto de bienestar; cabe imaginar que si la tarea de este mismo grupo de avezados pensadores hubiese sido definir el concepto de bienestar, lo hubieran hecho diciendo que es "el completo estado de salud física, mental y social y no sólo la ausencia de algún malestar". Así, en esta definición la palabra bienestar es usada como sinónimo de salud, por lo que incluye la idea que pretende definir.

Por la forma de enunciar esta definición, parece que la salud es equiparable a una vida placentera en lo físico, en lo mental y en lo social, como si el placer emanado de los sentidos corporales fuese el fin último que persigue una vida sana y, al construir la definición los autores se hubieran inspirado en la salud eterna del "cielo prometido" en vez de proponer una definición operativa para propósitos terrenales. Es, pues, más fácil censurar una definición de salud que construir otra, por eso puede ser de interés conocer el desarrollo histórico que ha tenido este concepto en distintas culturas, para identificar en ellas algunos elementos conceptuales que permitan una aproximación más universal al mundo interno que evoca esta palabra.

\section{El concepto de salud en el mundo antiguo}

En el Antiguo Egipto. A diferencia de los babilonios los egipcios vivían pensando en la muerte, como ilustra un breve fragmento literario: "...la muerte es hoy a mis ojos, como cuando uno desea ardientemente volver a su casa". Los hombres de las orillas del Río Nilo nacían de una compleja interacción de fuerzas materiales y espirituales dispuestas por los dioses, pero comprendían su vida terrenal como si fuese una imagen fugaz del espectro que sería su vida eterna. El cuerpo humano, sus órganos y sus instintos correspondían a lo que llamaban Khet, ente inserto en el mundo físico. Este cobraba vida gracias al $K a$, que era la fuerza vital con la que el hombre adquiría identidad; en ella residía la esencia íntima de lo que Freud llama ego. A esta potencia se superponía el $B a$ (súper ego) de origen divino, que por unión efectiva con su creador se convertía en Akha. A este conjunto de fuerzas y materia que daban forma a un sujeto se le asignaba un nombre, el cual correspondía a la expresión audible de su personalidad. ${ }^{3}$

En este reino sombrío, la enfermedad y la muerte eran condiciones inherentes a la naturaleza humana: la salud y la enfermedad sólo eran manifestaciones de un drama metafísico originado por causas externas. Se creía que la enfermedad y la muerte eran debidas a 
fuerzas extrañas, mediadas por objetos inanimados, fuesen éstos seres vivos o espíritus demoniacos. Creían que "el soplo de la vida entraba por la oreja derecha y el soplo de la muerte por la oreja izquierda"; con el soplo de la muerte se rompía la armonía entre la parte material y espiritual del hombre. Entre estos dos extremos, de la vida y de la muerte, la salud estaba subordinada a la interacción armónica de las fuerzas de la materia y del espíritu, en tanto que la gravedad de la enfermedad dependía del grado en que la armonía era trastocada.

En la Mesopotamia. Desde que el hombre tuvo la capacidad de razonar, la enfermedad -y no la salud- ha sido objeto de reflexión. En las culturas mesopotámicas que florecieron hace cuatro o cinco mil años, prevalecía la idea de que las enfermedades eran el castigo que los dioses imponían a los hombres que transgredían sus normas, aunque también algunas enfermedades podían ser causadas por maleficio de alguna persona o por el capricho de demonios errantes. Por eso, para recuperar la salud era necesario restablecer la armonía entre el dios ofendido y el hombre pecador, y según fuese la causa de la enfermedad el paciente era objeto de ritos religiosos o bien expuesto a exorcismos y magia. ${ }^{4}$ Aunque los conocimientos y creencias de estas culturas fueron asimilados, enriquecidos y aun superados por otros pueblos de las riveras del Mediterráneo, sus ideas ancestrales aún permanecen ocultas en el inconsciente colectivo: aún hay personas que consideran las enfermedades como castigo que los dioses imponen a los hombres o piensan que son producto del maleficio causado por algunas personas.

En China. Dentro de la filosofía China, caracterizada por asimilar elementos de las distintas corrientes que han florecido en su cultura milenaria, se considera que la salud -como todo lo que existe en el universoestá vinculada a un ente inmutable y eterno conocido como Tao. En el siglo VI a. C. prevalecía la idea de que la naturaleza estaba constituida por cinco elementos (madera, agua, fuego, tierra y metal) y era común la creencia de que todo en la naturaleza dependía de la interacción de dos fuerzas bipolares, del ying y del yang (yin-yang). Estos conceptos, y los de la vieja sabiduría preservada en el Libro de las Mutaciones, sirvieron a Lao Tse de fundamento al escribir su libro Tao-Te-King, que impulsó el taoismo. En esta doctrina el "Tao absoluto" que describe Lao Tse (sin describirlo), trasciende más allá de los conceptos conocidos desde la antigüedad como el "Tao del cielo" y el "Tao del hombre". En el Tao absoluto reconoce el principio fundamental de la virtud y la vida, y establece como meta suprema del taoísmo la inmortalidad corporal. Para lograr ese propósito considera necesario la obser- vancia de leyes morales, la exaltación mística y recursos mágicos, además de alimentar el cuerpo (yang-sing), alimentar el espíritu (yan-chen) y concentrarse en el Uno (cheu-yi). . $^{5}$

Conforme a esta ideología, la armonía del cuerpo y el espíritu se debe a dos elementos complementarios en el Tao: el yin y el yang. Del balance armónico de los atributos del yin (femenino, oscuro, pasivo, misterioso, demoniaco) y del yang (masculino, brillante, activo, claro, benéfico) depende la salud y la vida. Estos dos elementos se relacionan con cada una de las partes corporales mediante el $k^{\prime} i$-hiue, el sistema que distribuye en el cuerpo el soplo de energía vital $\left(k^{\prime} i\right)$ y la sangre (hiue); el K'i-hiue se vincula con elementos activos de la naturaleza (madera, agua, tierra, fuego y metal) manteniendo así la armonía del cuerpo con su medio externo. Del equilibrio entre el yin y el yang va a depender que el hombre goce de salud o padezca alguna enfermedad. El exceso del yin conduce a enfermedades agudas, febriles, secas, mientras que el exceso de yang da lugar a enfermedades crónicas, frías y húmedas. ${ }^{7}$

Es pertinente señalar que los elementos agua, fuego, madera, metal y tierra, no se contemplan en esta cultura sólo como parte de las cosas materiales, como entes inertes, sino como agentes dinámicos que actúan en todos los procesos que ocurren en la naturaleza. Son, pues, agentes activos que asocian al hombre con los puntos cardinales, con los cinco colores primarios, los cinco animales y las cuatro estaciones. El hombre, la tierra y el color amarillo -puesto que fue en el valle del Río Amarillo donde nació la civilización china- ocupan la posición central (figura 1); a partir del Centro se distribuyen los puntos cardinales en los que se agrupan el resto de los elementos: las estaciones del año, los colores y los animales míticos (el dragón, la tortuga, el tigre y el pájaro). El simbolismo de esta concepción esotérica, con interpretaciones profanas, es la que define el rumbo del hombre; sin embargo, la ventura o desventura de los seres humanos depende de la asociación equilibrada de estos elementos y de la armonía que mantengan las fuerzas del yin y el yang. ${ }^{8}$ En la India. La medicina Ayurveda (o saber sobre la longevidad) desarrollada entre el siglo VII y VIII a. C., aún preserva la idea de que para entender las enfermedades es preciso reconocer que los elementos esenciales del cuerpo humano son los mismos del macrocosmos: éter (o vacío), viento, fuego, agua y tierra. La combinación de estos elementos, en proporciones diferentes, es lo que da origen a los órganos corporales: el elemento que predomina en un órgano es el que define su función. Así, el éter predomina en los órganos huecos, como el intestino; el "viento", en los "so- 


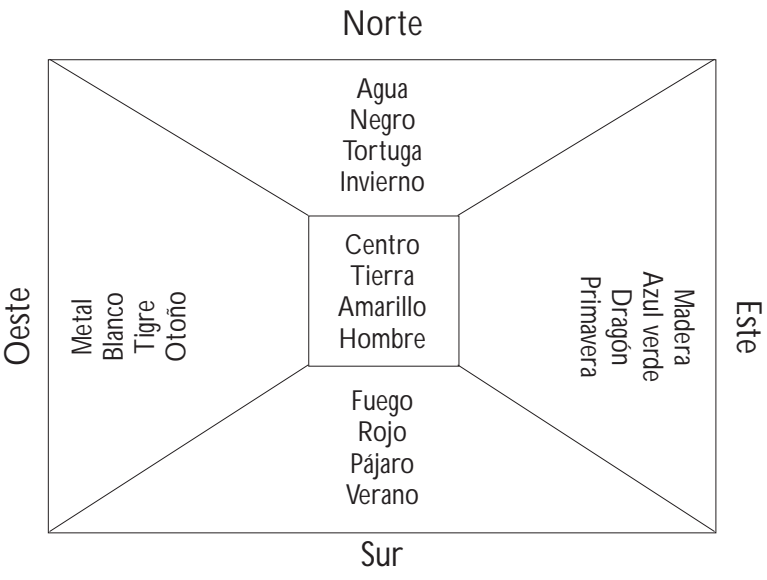

Figura 1. Cosmogonía de la China antigua que SITUABA AL HOMBRE EN EL CENTRO DEL COSMOS Y LO VINCULABA CON LOS "ELEMENTOS ACTIVOS" DE LA NATURALEZA, CON LOS PUNTOS CARDINALES, CON LAS ESTACIONES DEL AÑO, CON CINCO ANIMALES SAGRADOS Y CON LOS COLORES PRIMARIOS ${ }^{8}$

plos" que corren por el cuerpo; el agua, en los líquidos corporales, como la sangre; el fuego, en el calor interno del cuerpo; y la tierra, en los tejidos corporales y los músculos. Tres de estos elementos son los que desempeñan el papel más importante: el viento, el fuego y el agua; son los que sostienen las fuerzas que preservan la vida. Cuando en estos hay un desequilibrio, o perturbación, esto se traduce en tres "desordenes" o defectos (tridosha) que identifican a la enfermedad. En cambio, la salud radica en la armonía de estos elementos. ${ }^{9}$

En la cultura grecolatina. Apenas un siglo antes de surgir el taoísmo, este mismo concepto de armonía se encontraba vigente en la cultura helénica. Las ideas de Pitágoras (582-497 a. C.) de que el número marca el límite, el orden y la armonía del Cosmos (Kosmos) las aplicó también a los seres humanos. Pensaba que todo es número, todo es harmonía: los hombres, los órganos y los miembros corporales. La harmonía del todo y de sus partes es lo que genera en el hombre vida y salud; la desarmonía lleva a la enfermedad y a la muerte. Tenía el convencimiento de que la salud se encuentra en la medida, por lo que el precepto de mesura en todos los actos de la vida era una condición indispensable para conservar la armonía corporal, o para rescatarla si ésta se llegara a perder. ${ }^{10,11}$

En el último tercio del siglo VI a. C. Alcmeón de Crotona, estaba convencido también de que la salud del cuerpo y del espíritu dependía de la isonomía, entendida esta palabra como equilibrio, equidad, armonía o, en cierto sentido, la democracia de las fuerzas húmedo-seco, frío-caliente, amargo-dulce y de otras. La preminencia de una sola fuerza sobre la otra es causa de enfermedad. ${ }^{11,12}$ De acuerdo con los conceptos de este médico y filósofo el tratamiento de las enfermedades debe proveer la acción de la fuerza contraria a aquella cuyo exceso, o deficiencia, produce la enfermedad: frío contra calor, sequedad contra humedad, etcétera. ${ }^{12}$

Tiempo después, en la Magna Grecia, durante la primera mitad del siglo V a. C., Empédocles (490-430 a. C.) señalaba que los principios elementales del Cosmos eran fuego, aire, agua y tierra, los que eran también las sustancias esenciales del cuerpo humano. Anaximandro había atribuido a estos elementos la cualidad de ser, respectivamente, caliente (fuego), frío (aire), húmedo (agua) y seco (tierra), pensaba que la diferencia en la proporción en que intervienen estos elementos en las plantas, los animales y los hombres, era la característica genérica que los identificaba; con la particularidad de que aun entre ellos mismos hay algunas discrepancias en la proporción que guardan esas sustancias.

En esta cosmogonía, Empédocles asigna al amor y al odio un papel esencial; creía que la mezcla íntima que une a los cuatro elementos se daba porque el amor ha dominado al odio. Pensaba que la esfera primitiva del amor es la que mantiene la cohesión y la harmonía de las sustancias esenciales, pero cuando el nexo se pierde por predominio o por deficiencia (eklipsis) de alguna de ellas, la salud se altera. Consideraba que la respiración es el acto más elemental de la vida: con esta función se establece el vínculo entre los elementos primigenios y el medio externo. Para él, el vacío que ocurre parcialmente al fluir la sangre hacia los órganos es lo que atrae el aire al interior del cuerpo durante inspiración, pero luego el aire precisa ser expulsado al moverse la sangre hacia su lugar original durante la espiración. ${ }^{11-13}$

A los conceptos de Alcmeón, de que la salud es producto de la mezcla armoniosa de cualidades asimétricas, y a los de Empédocles, con respecto a la isonomía de los cuatro elementos, Pólibo ${ }^{14}$ (a quien se atribuye el libro "De la naturaleza del hombre", del Corpus hipocraticum) incorporó la vieja creencia de la participación de los humores corporales en el proceso de salud-enfermedad; este protomédico pensaba que el cuerpo humano contiene sangre, flema, bilis amarilla y bilis negra (y que) éstos son los elementos que entran en su constitución y explican sus dolores y su salud. La salud es, primariamente, el estado en el cual estas sustancias se encuentran, cada una, en su correcta 
proporción, en intensidad y cantidad, bien mezcladas. La enfermedad aparece cuando la cantidad de alguna se excede o hay deficiencia en la proporción de ella, o no se encuentra mezclada con las otras por estar separada del cuerpo. Es inevitable que no sólo la parte de donde se desprende la sustancia se vea afectada por enfermedad sino que también se altera aquella donde se encuentra en exceso, siendo causa de pena y dolor. Los humores difieren en su cualidad, de calor, frío, sequedad y humedad... cada uno posee su propia calidad; del equilibrio o desequilibrio de ellos, como respuesta a la combinación de sus cualidades (caliente, frío, húmedo y seco) y, de acuerdo con los elementos primarios de la naturaleza (aire, agua, tierra, fuego), es posible identificar si una persona goza de salud (eukrasia) o está enfermo (diskrasia)

Siete siglos después de haber nacido la doctrina hipocrática, durante el apogeo del imperio romano en el siglo II d. C., los estoicos, en voz de Marco Aurelio, ${ }^{15}$ pensaban que la naturaleza del universo dispone que el hombre enferme [..] a pesar de que el médico pretenda conducirlo a la salud, por eso, lo que le pase a cualquiera es porque se le ordena como a una más de las cosas subordinadas a la naturaleza [...] Aceptemos entonces alegremente lo que pase, por duro y desagradable que esto sea, con ello contribuiremos a la salud y bienestar del universo [...] Él es el Administrador de todo. De acuerdo con esta corriente filosófica, interpretada por el sentir de este emperador guerrero, lo que importa es la armonía del universo aunque ésta se logre a expensas de la salud o la vida de los seres humanos.

En ese mismo siglo Galeno reafirmó y divulgó la manera de pensar de Pólibo, enriqueció con ideas originales la doctrina hipocrática y con experiencias propias acrecentó los viejos conocimientos de la Escuela de Cos fundada por Hipócrates. Fue uno de los escritores médicos más prolíficos; sus numerosos textos médicos ejercieron una influencia significativa en el ejercicio de la medicina por 1500 años, cimentando la identidad de la medicina occidental.

\section{El concepto de salud de la Edad Media al siglo $X I X$}

Al desaparecer el Imperio Romano de Occidente, médicos árabes, persas y judíos, resguardaron e impulsaron los conocimientos de la medicina griega durante la Edad Media. Sigerist ${ }^{16}$ considera que la teoría de los cuatro humores ejerció entre los médicos mayor influencia en la Edad Media que en la época antigua, debido a que ilustraba de manera gráfica la interpretación filosófica de la salud y la enfermedad; señala que a partir del siglo XII, cuando se conoció en occidente la literatura árabe, estas ideas influyeron en el pensamiento médico y en el ejercicio de la medicina. Probablemente Rhazes, Alí Abbas y Avisena, en el este del mundo árabe, y en España, Abulcasis, Averroes, Avenzoar y el judio Moisés Ben Maimun, mejor conocido como Maimónides, tuvieron esta teoría como fundamento de su práctica médica.

Los médicos árabes y judios continuaron siendo líderes en la medicina occidental hasta la fundación de la Escuela (médica) de Salerno, la que retomó la idea grecolatina de fomentar la salud mediante preceptos higiénicos, lo cual divulgó en un manual de salud conocido como Regimen Sanitatis Salernitanum, publicado por primera vez en el siglo XIII; Sigerist ${ }^{2}$ considera que "la historia de la higiene desde el siglo XIII al siglo XIX podría ser escrita con sólo discutir los hechos a las ediciones de este texto". Cabe suponer que entre los médicos formados en ese lapso histórico permanecieron vigentes los conceptos hipocráticos de higiene, aunque se puede pensar que la opresión del régimen feudal, las epidemias recurrentes, las guerras, el hambre y otros infortunios, hicieron de la salud una entelequia. Todas estas calamidades contribuyeron a la expansión del cristianismo y con ello nació la firme creencia de que la salud, la enfermedad, el bienestar, la infelicidad y que todo lo concerniente al hombre es designio de Dios.

El concepto decimonónico de salud. Entre 1453 y 1789, lapso en el que convencionalmente los historiadores identifican la Edad Moderna, el saber y el ejercicio de la medicina continuaron sustentados en las ideas de Hipócrates y Galeno. Sin embargo, en el umbral del renacimiento, contagiado por los descubrimientos de nuevos continentes, por la revolución de las ideas y por el resurgimiento de las artes, Aurolus Filippus, Teofrasto, Bombasto de Hohenheim (1492-1541), conocido como Paracelso, ${ }^{17}$ intuyó otros horizontes en la medicina después de observar cierta analogía entre los procesos fisiológicos y patológicos con algunas reacciones químicas observadas en su rudimentario laboratorio alquimista. Se preguntaba si era posible explicar los mecanismos de las enfermedades en términos químicos. Así, poco a poco fue dando forma a una teoría en la que incorporó conceptos de la química: creía que en los órganos corporales se encontraban tres principios físicos: el combustible, el volátil y el incombustible. A estos principios les dio el nombre simbólico de azufre (porque arde), mercurio (porque echa humo) y sal (porque permanece en las cenizas). Supuso que estas sustancias eran los elementos químicos 
que integran el cuerpo humano, pero además supuso la existencia de un principio vital al que llamó archaeous.

Para explicar el mecanismo por el cual los hombres adquieren una enfermedad, Paracelso desarrolló un sistema integrado por cinco entes (ens). Acorde con estas ideas, estaba convencido que para que el hombre recupere o conserve su salud es preciso un "alquimista" hábil que separe los principios buenos de los malos que hay en el medio ambiente (ens veneni), pero además requiere contar con el favor de los astros (ens astrale), con la constitución natural (ens naturale) de cada ser humano y con su espiritu (ens spirituale), todo esto en consonancia con el pensamiento escolástico que dominó el pensamiento durante el medioevo: con la anuencia de Dios (ens Dei), de quien depende la alquimia de la salud que preserva la vida.

Tres siglos después de que William Harvey (15781657) desarrolló las investigaciones que permitieron comprender la circulación de la sangre, en el siglo XIX Claudio Bernard introdujo la medicina al mundo de la ciencia. Entre las reflexiones acerca de la salud que documenta en su libro: "Introducción al estudio de la medicina experimental", está lo siguiente: La condición necesaria para la vida (sana) no se encuentra ni en el organismo ni en el ambiente externo, sino en ambos. Si suprimimos o alteramos alguna función del organismo, la vida cesa, aun cuando el ambiente permanezca intacto; por otro lado, si modificamos los factores del ambiente que se asocian con la vida, ésta puede desaparecer, aun cuando el organismo no haya sido alterado [...] En los seres vivos el ambiente interno, que es producto del funcionamiento del organismo, preserva la relación necesaria de intercambio y equilibrio con el ambiente externo. ${ }^{18}$

Desde la perspectiva de Bernard, la salud depende del funcionamiento armónico de los órganos y los sistemas corporales. Señala que la "relativa constancia" del medio interno, a pesar de las variaciones del medio externo, se traduce en el funcionamiento de los órganos y sistemas corporales dentro de un margen de normalidad. Por eso consideraba que la salud del hombre depende de una "relación necesaria de intercambio y equilibrio" al interactuar con fuerzas físicas, objetos materiales, sustancias químicas y seres biológicos del ambiente externo.

\section{El concepto contemporáneo de salud}

Probablemente Perkins ${ }^{19}$ se inspiró en los conceptos de Cl. Bernard al definir, en 1938, la salud como "un estado de relativo equilibrio de la forma y función corporal, que resulta del ajuste dinámico del organismo ante las fuerzas que tienden a alterarlo. Y añadía, "no es, pues, el resultado de la interrelación pasiva entre las sustancias del organismo y los factores que pretenden romper la armonía con el medio externo, sino la respuesta activa de las fuerzas corporales que funcionan para establecer los ajustes para preservar la vida".

Con esta misma idea Dubos ${ }^{20}$ interpretó el concepto de salud en un sentido ecológico: pensaba que la vida implica la interacción y a la vez la integración de dos ecosistemas: el medio interno y el medio externo. Por un lado el organismo, cuyas células, fluidos y estructuras tegumentarias están relacionadas entre sí mediante una compleja red de mecanismos de equilibrio, y por el otro, el medio externo está sujeto a cambios que acontecen en ocasiones de manera impredecible; muchos de estos cambios que ocurren en el ambiente externo pueden causar efectos dañinos en el hombre, por lo que en el organismo se generan cambios adaptativos para funcionar de manera eficiente y conservar la vida. Cualquier factor que rompa el equilibrio entre estos dos sistemas ecológicos puede ser causa de enfermedad.

Hasta aquí, las definiciones de salud habían ubicado su interés en el hombre individual olvidándose de la salud colectiva: la de las poblaciones humanas, por eso es interesante rescatar el hecho de que un destacado fisiólogo, preocupado por explicar los fenómenos vitales del hombre, haya dejado escapar su imaginación para tratar de entender las circunstancias que actúan en la salud de las colectividades. En la década de los años treinta del siglo XIX Walter B. Cannon, ${ }^{21}$ quien llamó homeostasis biológica la relativa estabilidad del medio interno descrito por Bernard, extrapoló este concepto a la población denominándolo homeostasis social; pensaba que en ella salud depende del equilibrio armónico de todos los elementos que dan cohesión a la sociedad. Para algunos, la opinión de Bernard es una simplificación poco afortunada ya que omite destacar la importancia que tiene la estructura política en la salud de la población: en ella recae la responsabilidad de procurar el bienestar de la sociedad, tal como Rudolf Virchow afirmaba "[El Estado] debe ayudar a todos a vivir una vida sana. Esto se desprende sencillamente de la concepción del Estado como unidad moral de todas las personas que lo constituyen y de la obligación de solidaridad universal" ${ }^{22}$

El hecho de recordar las ideas de Virchow obedece a que en años recientes han cobrado importancia los programas de promoción de la salud y por ende la responsabilidad del estado en procurar lo que Cannon denomina "homeostasis social"; en las conclusiones de la reunión convocada por la OMS para la 
Promoción de la Salud en el Siglo XXI, que tuvo lugar en Jakarta en 1997, se establece que "Los requisitos para la salud [de la población] son: paz, vivienda, educación, seguridad social, relaciones sociales, alimentación, ingreso económico, "empoderamiento de la mujer", un ecosistema estable, uso de recursos sostenibles, justicia social, respeto por los derechos humanos y equidad".${ }^{23}$ En la medida en la que se alcancen estos requisitos de convivencia social cabe esperar que la población goce de salud y bienestar.

\section{El concepto de salud en la segunda mitad del siglo $X X$}

En 1941 Sigerist definía la salud diciendo que "salud no es simplemente la ausencia de enfermedad, es algo positivo, una actitud gozosa y una aceptación alegre de las responsabilidades que la vida impone al individuo"; esta definición tal vez inspiró a Sampar para construir la que propuso a la OMS en 1946 y que aparece en su Carta Constitutiva, a la cual ya se ha hecho referencia. ${ }^{24}$ Veinte años después, en 1966, Georges Canguilhem añadió a su libro "Nuevas reflexiones relativas a lo normal y patológico"; entre ellas hace algunas observaciones y agrega algunos conceptos con los que hace un esbozo de lo que para él es la salud.

Imbuido en la corriente vitalista, según opinión expresada por Pérez Tamayo, ${ }^{25}$ Canguilhem sostiene que "la salud es la posibilidad de superar la norma que define lo momentáneamente normal, la posibilidad de tolerar infracciones a la norma habitual e instituir normas nuevas en situaciones nuevas" ${ }^{26}$ Continúa con una serie de argumentos en los que plantea su idea personal acerca de lo normal y lo normativo para agregar que "la salud es un conjunto de seguridades en el presente y aseguramientos para el futuro" en lo biológico y en lo psicológico, para regular las posibilidades de reacción del individuo. Señala que el hombre sano mide su salud por su capacidad para superar las crisis orgánicas e instaurar un nuevo orden; resume sus reflexiones, mencionando que "El hombre sólo se siente en buen estado de salud -tal es la salud- cuando más que normal -es decir adaptado al medio ambiente y a sus exigencias- se siente normativo, apto para seguir nuevas normas de vida". Termina sus argumentos con el matiz personal que sólo ese médico y filósofo puede dar a sus ideas, y señala "que la salud es una manera de abordar la existencia sintiéndose no sólo poseedor sujeto y su mundo y sobre esto versa la observación del médico".

\section{Conclusiones}

Por la evolución histórica del pensamiento legado por las culturas que dan identidad al mundo actual, se sabe que antes de que los filósofos reflexionaran sobre la razón y destino del hombre cavilaron acerca de los componentes del cosmos y las sustancias esenciales del cuerpo humano. Casi todas las grandes culturas coinciden en señalar como elementos primigenios del organismo: el agua, el fuego, el aire (viento) y la tierra, unidos por la armonía del yin y el yang (China), el equilibrio de fuerzas vitales (India) y la equidad (isonomía) entre las sustancias que integran la materia humana (Grecia) (cuadro I). Tal parece que la salud gira en torno a los conceptos de armonía, de equilibrio y de equidad, y lo opuesto se atribuye a la enfermedad. El problema para comprender cabalmente el significado de esa palabra radica en identificar la línea intangible que separa estos conceptos bipolares.

Para Aristóteles ${ }^{27}$ "de la salud no resultan efectos contrarios, sino solamente saludables"; considera que una de las reglas normativas de la ética es la del "justo medio": su aplicación permite adivinar los límites intangibles que separan conceptos abstractos como la salud y la enfermedad. Juliana González ${ }^{28}$ piensa que

\section{LA NATURALEZA DEL MACROCOSMOS Y DEL MICROCOSMOS (HOMBRE), Y EL CONCEPTO DE SALUD ENFERMEDAD EN DISTINTAS CULTURAS}

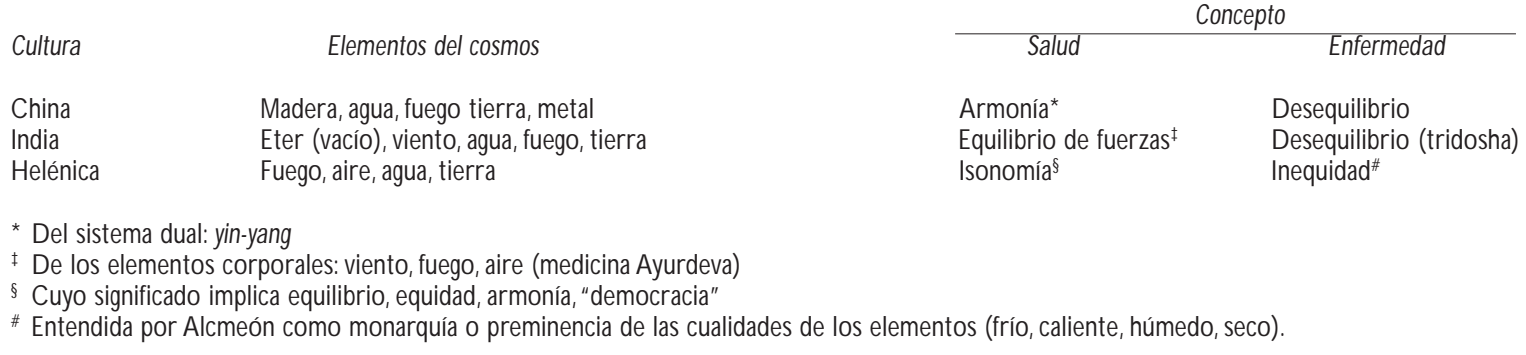


el "justo medio" "es el punto de equilibrio entre las fuerzas: la armonía entre lo divino y lo humano; (añade) ... La salud nunca fue vista (entre los griegos) al margen de la armonía y de "la gran salud del universo", de su orden secreto, de los Fines primordiales de la naturaleza", de tal manera que conservar o restablecer la interacción armónica entre las fuerzas de la materia y el espíritu, son tareas imprescindibles para su salud: el universo es el Administrador de todo, según pensaba Marco Aurelio. ${ }^{15}$

Una de las particularidades de la medicina helénica es considerar el cuerpo humano de manera integral: relaciona el todo con cada una de sus partes y con el entorno mismo; parece que esta percepción daba sentido a los conceptos de salud y enfermedad. Consideraban la salud como un fenómeno de armonía y proporción, no como producto de las cualidades de la materia, sino como equilibrio de las fuerzas y por la mezcla de los componentes corporales, y sus interrelaciones, y todo esto como respuesta del organismo a los estímulos del ambiente. Contemplaba al hombre dentro de su entorno físico y social, lo que explica porque ubicaban la salud y la enfermedad en este ámbito, pero sin desatender las situaciones particulares, siempre cambiantes. Por eso se piensa ${ }^{28}$ que uno de los legados más valiosos de la medicina griega es lo concreto, en su connotación latina (concretus: lo que crece junto, no lo aislado o separado), que permite adivinar el "juego inmenso de las relaciones del sujeto y su mundo, sobre esto versa la observación del médico".

En esta perspectiva histórica parece que desde la antigüedad predomina la idea de que la salud es una condición que traduce el "equilibrio dinámico" entre el hombre y su ambiente. Hay ahora cabal convicción que desde antes de la etapa embrionaria el hombre sostiene una perenne confrontación con innumerables factores: unos favorables, otros adversos; su salud depende del ajuste dinámico de su organismo al entorno.

\section{Referencias}

1. Márquez F. Frases celebres. Madrid: ED IMAT libros, 2000:324.

2. Sigerist HE. Health.J Public Health Pol 1996;17:204-234.

3. Sendrail M. Historia cultural de la enfermedad. Madrid: Espasa-C alpe, 1983:57-76.
4. Zaragoza JR. La medicina de los pueblos mesopotámicos. En: Entralgo PL, ed. H istoria universal de la medicina. Tomo 1. Barcelona: Salvat, 1976: 67-93.

5. Huard P,W ong M. La medicina C hina. En: Entralgo PL, ed. Historia universal de la medicina. Tomo 1. Barcelona: Salvat, 1976:159-195.

6. W ilhelm R. Enseñanzas de Lao Tse. En:Tse L, 0 kakura K, ed. Maestros orientales. Tao Te King/ El Libro del té. Barcelona: Edicomunicación, 1999: p 6-34.

7. Colección Wellcome sobre Historia de la medicina, museo Príncipe Alberto. Londres, 1997.

8. Schafer EH . La China antigua. Madrid:Time-Life international (Holanda), 1978:101-110.

9. Riviere JR. La medicina en la antigua India. En: Entralgo PL. Historia universal de la medicina. (Tomo 1). Barcelona: Salvat, 1976:129-158.

10. Brun J. Los presocráticos. México, D.F.: Conaculta, 1995.

11. Lasso de laVega JS. Pensamiento presocrático y medicina. En: Entralgo L. Historia universal de la medicina. Barcelona: Salvat, 1982;Tomo 2:37-71.

12. Heráclito, Parménides, Empédocles. Textos presocráticos. Barcelona: Edicomunicación, 1999:9-69.

13. Entralgo PL. La medicina hipocrática. Historia universal de la medicina Barcelona: Salvat, 1982;Tomo 2:72-117.

14. Lloyd GER, ed. Hippocratic writings. Harmondsworth: Pelican Books, 1978:260-271.

15. Marcus Aurelius. Meditations. Harmondsworth: Peguin Books, 1997: 45-46.

16. Sigerist HE. Civilización y enfermedad 1a ed. México, D.F.: Fondo de Cultura Económica, 1987:193-210.

17. Paracelso. 0 bras completas (O pera omnia) México, D.F.: CIN AR Editores, 1994:193-210.

18. Bernard C. Introducción al estudio de la medicina experimental. México, D.F.: Universidad N acional Autónoma de México, 1994:197-201.

19. Perkins W H. C ause and prevention of disease. Philadelphia (PA): Lea and Febiger, 1938.

20. Dubos R. El hombre en adaptación. México, D.F.: Fondo de Cultura Económica, 1975:303-306.

21. Cannon W B. La sabiduría del cuerpo. México, D.F.: Editorial Séneca, 1941:332-352.

22. Alleyne GAO . Promoción de la salud en las A méricas. En: Informe del Director -2001 W ashington, D.C .: O ficina Sanitaria Panamericana, 2001. 23.W orld Health 0 rganization. The Jakarta declaration on health promotion into XXI C entury: casa ed. Jakarta, July, 1997.

24. Piedrola G il. La salud y sus determinantes. Concepto de medicina preventiva y salud pública. En: Medicina preventiva y salud pública $10^{0} \mathrm{ed}$. Barcelona: Masson, 2001:3-14.

25. Pérez-Tamayo R.Tríptico. México, D.F.: El Colegio N acional, 1982: 15-41.

26. C anguilhem G. Lo normal y lo patológico 7ạ ed. México, D.F.: Fondo de Cultura Económica 1986:137-154.

27. A ristóteles: Ética nicomaquea. Política. "Sepan cuantos..." N 0. 70. México, D.F.: Editorial Porrúa, 1999:58-73.

28. González J. El ethos, destino del hombre. México, D.F.: Fondo de Cultura Económica, 1996:79-91. 